\title{
Pyrosequencing of supra- and subgingival biofilms from inflamed peri-implant and periodontal sites
}

Simone Schaumann ${ }^{1}$, Ingmar Staufenbiel ${ }^{2}$, Ralph Scherer ${ }^{3}$, Markus Schilhabel $^{4}$, Andreas Winkel ${ }^{1}$, Sascha Nico Stumpp ${ }^{1}$, Jörg Eberhard ${ }^{5^{*}+}$ and Meike Stiesch ${ }^{1 \dagger}$

\begin{abstract}
Background: To investigate the microbial composition of biofilms at inflamed peri-implant and periodontal tissues in the same subject, using $16 \mathrm{~S}$ rRNA sequencing.

Methods: Supra- and submucosal, and supra- and subgingival plaque samples were collected from 7 subjects suffering from diseased peri-implant and periodontal tissues. Bacterial DNA was isolated and 16S rRNA genes were amplified, sequenced and aligned for the identification of bacterial genera.

Results: 43734 chimera-depleted, denoised sequences were identified, corresponding to 1 phylum, 8 classes, 10 orders, 44 families and 150 genera. The most abundant families or genera found in supramucosal or supragingival plaque were Streptoccocaceae, Rothia and Porphyromonas. In submucosal plaque, the most abundant family or genera found were Rothia, Streptococcaceae and Porphyromonas on implants. The most abundant subgingival bacteria on teeth were Prevotella, Streptococcaceae, and TG5. The number of sequences found for the genera Tannerella and Aggregatibacter on implants differed significantly between supra- and submucosal locations before multiple testing. The analyses demonstrated no significant differences between microbiomes on implants and teeth in supra- or submucosal and supra- or subgingival biofilms.
\end{abstract}

Conclusion: Diseased peri-implant and periodontal tissues in the same subject share similiar bacterial genera and based on the analysis of taxa on a genus level biofilm compositions may not account for the potentially distinct pathologies at implants or teeth.

Keywords: Deep-sequencing, 16S rRNA sequencing, Diseased peri-implant tissues, Diseased periodontal tissues, Supragingival plaque, Subgingival plaque, Biofilm, Microbiology

\section{Background}

Dental implants are commonly used to replace missing teeth in partially edentulous or edentulous patients. Inflammation of the peri-implant soft and hard tissue is the most frequent adverse event and may compromise the long-term stability of osseointegrated implants. While periimplant mucositis affectes only soft tissues, peri-implantitis also involves the supporting bone. The prevalence of periimplantitis during 5-10 years after successful osseointegration seems to be of the order of $10 \%$ of implants and $20 \%$ of patients [1].

\footnotetext{
* Correspondence: Eberhard.joerg@mh-hannover.de

${ }^{\dagger}$ Equal contributors

${ }^{5}$ Peri-implant and Oral Infections, Department of Prosthetic Dentistry and Biomedical Materials Science, Hannover Medical School, Carl-Neuberg-Strasse 1, 30625 Hannover, Germany

Full list of author information is available at the end of the article
}

Accepted risk factors for peri-implant related diseases are poor oral hygiene, a history of periodontitis and cigarette smoking [2]. Biofilms have been described in detail by using hybridization techniques in peri-implantitis [3-6] and recently by high-throughput sequencing techniques in failing implants [7-9]. Supra- and submucosal biofilms on implants in individual subjects have not been described by using high-throughput sequencing techniques, although it has been shown that the composition of supragingival biofims significantly affects subgingival biofilm formation [10-12]. In consequence, supramucosal biofilms may also determine the composition of the submucosal microflora. The diverse surface properties (chemical composition, surface roughness, surface free energy) and tissue architecture at implants and teeth may affect bacterial adhesion and growth of biofilms as well [13] and 
may account for the proposed differences in inflammatory response at implants and teeth [14].

Therefore the aim of the following study was to further characterise the microbial composition of supra- and submucosal, repectively supra- and subgingival plaques at diseased implants and teeth.

\section{Methods}

\section{Subject selection}

Subjects included in the study had at least $\geq 30 \%$ sites with $\mathrm{PD} \geq 4 \mathrm{~mm}$ and evident radiographic bone loss. All patients were partially edentulous (not fewer than 8 teeth), with at least 1 functioning oral implant restored with crowns or prostheses. Inclusion criteria were: (A) one implant and teeth showing signs of active inflammation (tissue with manifest signs of inflammation (redness and swelling), bleeding on probing (BOP) and pocket depth (PD) $\geq 4 \mathrm{~mm}$ in at least one site and evidence of radiographic bone loss), (B) implants had to be functioning for at least 1 year. Exclusion criteria were: (A) any peri-implant or periodontal treatment 6 months before sampling. (B) systemic diseases such as diabetes mellitus, (C) smoking, (D) antibiotic or immunosuppressant medication within the previous 3 months.

A comprehensive medical history was recorded, followed by clinical and radiographic examination. Informed consent was obtained and the study was approved by the local Ethics committee of Hannover Medical School (no. 4348).

\section{Clinical examination}

Two experienced dentists examined all subjects. Pocket depth was measured using a pressure calibrated periodontal probe (Hawe Click-Probe, Kerr Hawe SA, Bioggio, Switzerland). Probing depth was measured to the nearest millimeter on the scale. Bleeding on probing was assessed after probing using a dichotomous measure. All measurements were performed on 4 sites of all implants and teeth. Plaque deposits were recorded (presence/absence) without staining, using a modified Approximal Plaque Index (API) [15].

\section{Sample collection}

In each subject, the implant and the tooth with the deepest depths were chosen for plaque collection. After isolating the sampling area with cotton rolls and gentle drying with an air syringe, 2 sterile endodontic paper points (Absorbent Paper Points, VDW GmbH, Munich, Germany) were used supramucosally or supragingivally to collect the biofilms. Subsequently, the residual supramucosal and supragingival plaques were completely removed with a dental scaler. Two sterile paper points were then placed submucosally or subgingivally. The samples were pooled separately for every implant, tooth and location and were placed in $2 \mathrm{ml}$ cryotubes (Eppendorf, Hamburg, Germany) and frozen immediately at $-80^{\circ} \mathrm{C}$ before processing.

\section{DNA extraction and sequencing DNA isolation}

Paper points used for sampling were treated with $360 \mu \mathrm{l}$ lysozyme solution for $30 \mathrm{~min}$ at $37^{\circ} \mathrm{C}(20 \mathrm{mg} / \mathrm{ml}$ lysozyme, $20 \mathrm{mM}$ TrisHCl, $2 \mathrm{mM}$ EDTA, $1.2 \%$ Triton X100, pH 8.00 ), followed by proteinase $\mathrm{K}$ digestion for $30 \mathrm{~min}$ at $56^{\circ} \mathrm{C}$ in $400 \mu \mathrm{l}$ buffer AL (Qiagen, Hilden, Germany). Enzymes were inactivated by heating to $95^{\circ} \mathrm{C}$ for $15 \mathrm{~min}$. Sterile $0.5 \mathrm{~mm}$ glass beads (Roth, Karlsruhe, Germany) were added and bacterial cells were disrupted by vigorous shaking (6500 rpm, 3 x 20s, 15s break) with a Precellys 24 bead mill (Bertin Technologies, Montigny-le-Bretonneux, France). Subsequently, total DNA was purified with the QIAamp DNA Mini Kit (Qiagen, Hilden, Germany) according to the manufacturer's protocol for gram-positive bacteria (QIAamp DNA Mini and Blood Mini Handbook, Third Edition, Appendix D).

\section{$16 \mathrm{~S}$ rDNA amplification and sample preparation}

From each sample, an approximately $550 \mathrm{bp}$ fragment of the 16S rRNA gene was amplified using the broad range primers 27f (5'-AGAGTTTGATCMTGGCTCAG-3') and 521r (5'-ACCGCGGCTGCTGGCAC-3'; both Eurogentec, Seraing, Belgium). The primers targeted conserved DNA sequences flanking the V1 and V3 hypervariable regions within the 16S rRNA gene. PCR was performed on a TProfessional thermocycler (Biometra, Göttingen, Germany) in a total reaction volume of $50 \mu$ l. The PCR mix contained approximately $20 \mathrm{ng}$ of template DNA, $200 \mathrm{nM}$ of each primer, 1x PCR buffer (including $1.5 \mathrm{mM}$ magnesium chloride; Qiagen, Hilden, Germany), 1.5U HotStar Taq polymerase (Qiagen, Hilden, Germany), $200 \mathrm{mM}$ of each dNTP (Roth, Karlsruhe, Germany) and PCR-grade water (Roche, Penzberg, Germany). PCR conditions were as follows: Initial denaturation at $95^{\circ} \mathrm{C}$ for $15 \mathrm{~min} ; 32$ amplification cycles consisting of denaturation at $94^{\circ} \mathrm{C}$ for $1 \mathrm{~min}$, annealing at $52^{\circ} \mathrm{C}$ for $40 \mathrm{~s}$, elongation at $72^{\circ} \mathrm{C}$ for $1 \mathrm{~min}$; final extension at $72^{\circ} \mathrm{C}$ for $10 \mathrm{~min}$. PCR reactions were separated on a 1.0\% agarose gel (Agarose MP; AppliChem, Darmstadt, Germany) and purified using the QIAquick Gel Extraction Kit (Qiagen, Hilden, Germany). The purified amplicons of each sample were used as template for a second PCR step with the primer $27 \mathrm{f}-\mathrm{AdaB}$ (5'-CCTATCCCCTGTGTGCCTTGGCAGTCTCAGAGA GTTTGATCMTGGCTCAG-3') and an individual reverse primer 521r-MID_X (5'-CCATCTCATCCCTGCGTGTC TCCGACTCAGXXXXXXXXXXXACCGCGGCTGCTGG CAC-3'; XXXXXXXXXXX = unique MID-tag) containing a unique Multiplex-Identifier (MID) barcode sequence. Amplification chemistry was the same as described above, however, 100 ng of template DNA were used per reaction, 
the annealing temperature was raised to $67^{\circ} \mathrm{C}$ and the cycle number was reduced to 15 . PCR reaction products were purified by agarose gel electrophoresis and extracted as described before. The DNA concentrations were determined using the AccuBlue ${ }^{\mathrm{Tm}}$ High Sensitivity dsDNA Quantitation Kit (Biotium, Hayward, USA) in combination with a BioTekSynergy II fluorescence reader (BioTek, Bad Friedrichshall, Germany). Subsequently, the samples were mixed in an equimolar ratio and further processed according to the manufacturer's instruction for the Titanium Library Preparation Kit (Roche, Penzberg, Germany). Pyrosequencing was performed on a GS FLX sequencer (Roche, Penzberg, Germany).

\section{Bioinformatics}

\section{Sequence processing}

Qiime software version 1.6 [16] was used for preprocessing, the identification of operational taxonomic units (OTU), the taxonomic assignment and the community structure comparisons. In the preprocessing step, every 454-read was removed if (a) the number of base pairs was $<200$ or $>550$, (b) the quality score was $<25$, (c) the number of ambiguous bases was $>6$, (d) there was a primer mismatch, (e) the number of errors in barcode were $>1.5$, or (f) a homopolymer run was $>6$. In addition to these quality filtering steps, a denoising step of the sequences was performed [17] with the "denoise_wrapper"-script in qiime. Chimeric sequences were removed using ChimeraSlayer with the qiime default settings after OTU-picking and taxonomic assignment.

\section{OTU assignment and taxonomic classification}

The sequences were assigned to OTUs with the uclust method in qiime with a similarity threshold of 0.97 , which corresponds to genus level OTUs. For the following taxonomic assignment, we used the blast method in qiime with the greengenes 12_10 release with 97\% OTUs as the reference database. In addition, genera were categorized according to their Gram staining based on Bergey's Manual of Systematic Bacteriology.

\section{Statistical analyses}

The OTU-table created by qiime after denoising and chimera checking was imported into the statistical programming language $R$ [18] using the Bioconductor [19] package phyloseq [20]. The following graphical analyses were also performed using the phyloseq package and were created for (a) the whole data set, (b) the implant subset and (c) the tooth subset. The taxonomic rank used for the following analyses was the genus level. First, heat maps for the 50 most abundant bacteria were created. Second, Principal Coordinate Analyses (PCoA) of UniFac distances were calculated and plotted. The inferential statistical analysis was calculated with the Bioconductor package edgeR [21]. Therefore log Fold-Changes and corresponding multiplicity- adjusted $\mathrm{p}$ values were estimated from separate generalized linear models for every genus with patient as covariate and considering the paired design character. Biodiversity was calculated using the Shannon-Diversity Index [22].

\section{Results}

\section{Clinical data}

Seven subjects ( 2 males, 5 females, mean age $60.1 \pm 9.8$ years) were eligible for the study between August and October 2010 at Hannover Medical School, Department of Prosthetic Dentistry and Biomedical Materials Science. Individual data and full-mouth scorings of all patients are summarized in Table 1. All implants investigated had been functioning for an average of $11.6 \pm 5.5$ years. Clinical signs of inflammation were apparent at investigated implants (PD $4.9 \pm 1.2 \mathrm{~mm}$, BOP $39.9 \pm 34.9 \%$ ) and teeth (PD $4.1 \pm 1.2$ $\mathrm{mm}$, BOP $35.7 \pm 31.8 \%)$. Differences between the clinical recordings at implants and teeth were not significant (Table 1).

\section{Supra- and subgingival microbiomes}

28 supra- and subgingival samples from 7 patients were analyzed and yielded a total of 43734 chimera-depleted, denoised sequences representing 1 phylum, 8 classes, 10 orders, 44 families and 150 genera (Additional file 1). On implants, these sequences represented the families Porphyromonadaceae, Lachnospiraceae, Streptococcaceae and genera Rothia, Actinomyces, Paenibacillus, Microbacterium, Pseudoramibacter,

Table 1 Subject characteristics

\begin{tabular}{ll}
\hline Study population & \\
\hline Number of patients & 7 \\
Gender (male/female) & $2 / 5$ \\
Age (years) & $60.1 \pm 9.8$ \\
Implant longevity (years) & $11.6 \pm 5.6$ \\
Number of Implants per patient (n) & $4.7 \pm 3.6$ \\
Number of remaining teeth per patient (n) & $16.7 \pm 7.3$ \\
Full-mouth scores & $61.3 \pm 28.8$ \\
Plaque index, API (\%) & $22.1 \pm 16.2$ \\
BOP (\%) & $68.1 \pm 15.5$ \\
Number of periodontitis affected teeth per patient (\%) \\
Scores at sampled sites & \\
Implants & $35.7 \pm 37.8$ \\
Plaque index (\%) & $39.3 \pm 34.9$ \\
BOP (\%) & $5.0 \pm 1.3$ \\
PD (mm) & \\
Teeth & $28.6 \pm 39.3$ \\
Plaque index (\%) & $35.7 \pm 31.8$ \\
BOP (\%) & $4.1 \pm 1.3$ \\
PD (mm) & \\
\hline
\end{tabular}

Data are presented as means and standard deviations.

API, Approximal Plaque Index; BOP, bleeding on probing; PD, probing depths. 
Leptotrichia, Parascardovia, Tannerella, Granulicatella, Tessaracoccus, Clostridium, Aeromonadales, Veillonella, Capnocytophaga, Prevotella, TG5, Fusobacterium, Exiguobacterium, Enterococcus, Porphyromonas, Streptococcus at implants. On teeth, the sequences represented the families Coriobacteriaceae, Rs-045, Veillonellaceae, Neisseriaceae, and the genera Mogibacterium, Porphyromonas, Tannerella, Aggregatibacter, Treponema, Capnocytophaga, Lactococcus, Granulicatella, Enterococcus, Exiguobacterium, Atopobium,
Veillonella. On implants and teeth, the above-mentioned bacteria accounted for $>90 \%$ of all sequences.

In supramucosal or supragingival plaques on implants and teeth, the most abundant taxa were Streptococcacea, Rothia, and Porphyromonas. In submucosal plaques at implants, the most abundant taxa found were Rothia, Streptococcaceae and Porphyromonas. The most abundant subgingival bacteria on teeth were Prevotella, Streptococcaceae and TG5 (Figure 1a, b).

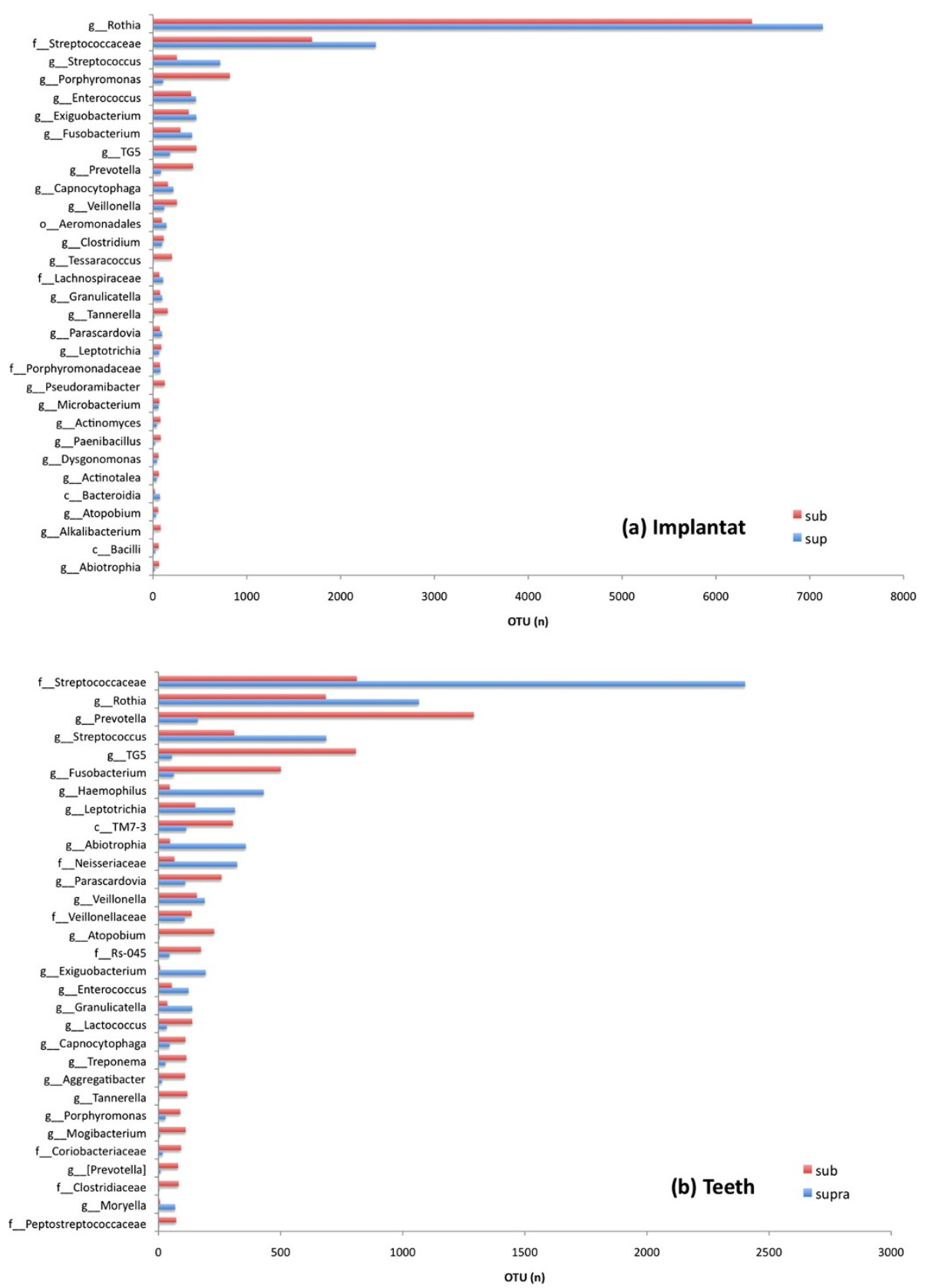

Figure 1 Detection frequency of taxa found in inflamed peri-implant and periodontal sites. (a) Distribution of taxa in supra- and submucosal biofilms from inflamed implants and (b) taxa in supra- and subgingival biofilms of teeth affected by periodontitis. The listed genera (g), families (f) and classes (c) represents $90 \%$ of all sequences found. 


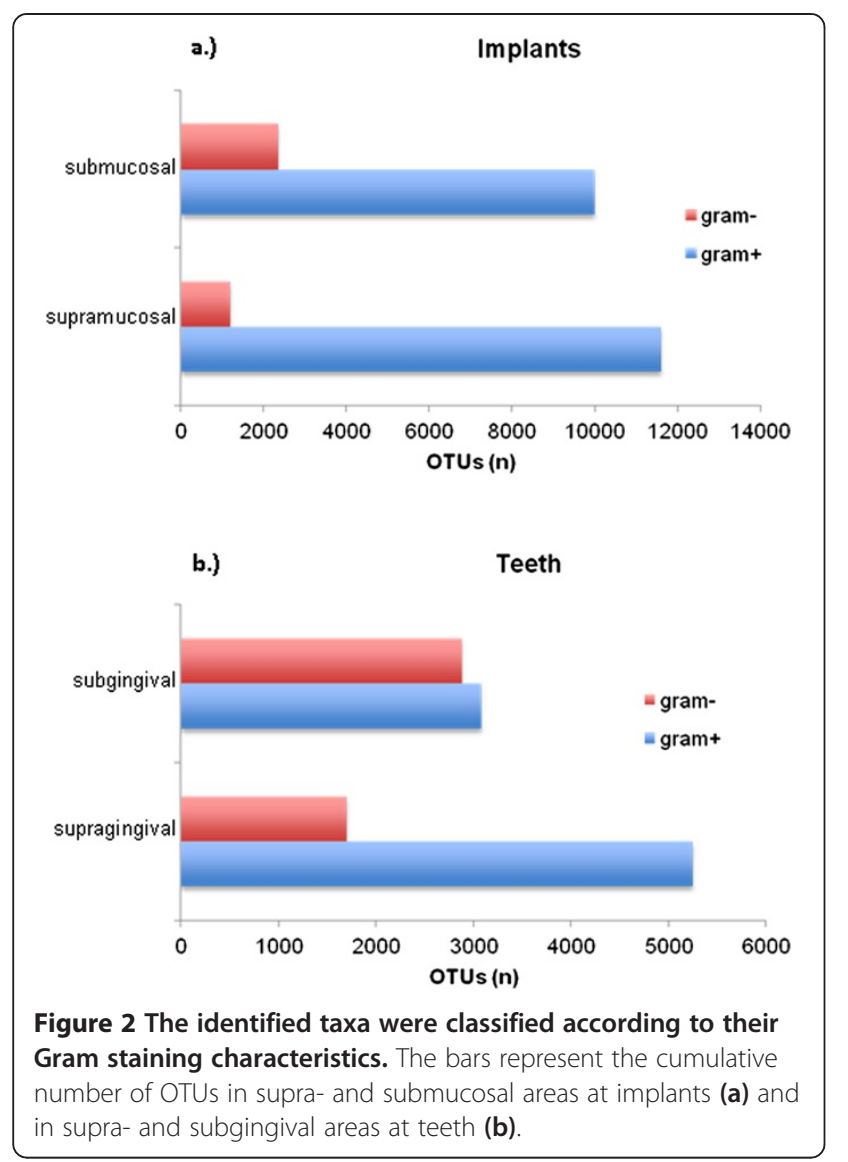

The statistical analysis showed significant differences between supra- and submucosal plaque on implants for the genus Tannerella $(\mathrm{p}=0.0067)$ and nearly significant differences for the genus Aggregatibacter ( $\mathrm{p}=0.056$ ). After correction for multiple testing, these differences were no longer significant.

\section{Gram stain categories}

The Gram stain categories on implants and teeth are presented in Figure 2a and b. In general, Gram-positive bacteria were more prevalent than Gram-negative bacteria in all samples. On implants, Gram-positive bacteria were predominately found in supra- and submucosal samples. In supragingival samples of teeth, Gram-positive bacteria were more frequent than Gram-negative bacteria, but in subgingival plaque samples the abundances of Gram-positive and Gram-negative bacteria were similar. On implants and teeth, the number of Gram-negative bacteria were greater at submucosal and subgingival locations than at supramucosal and supragingival sites.

\section{Principal coordinate analysis (PCoA)}

The Principal Coordinate Analysis (Figure 3) of weighted UniFac distances revealed no distinct partitioning of the bacterial communities associated with implants or teeth ( $\mathrm{p}>0.01)$.

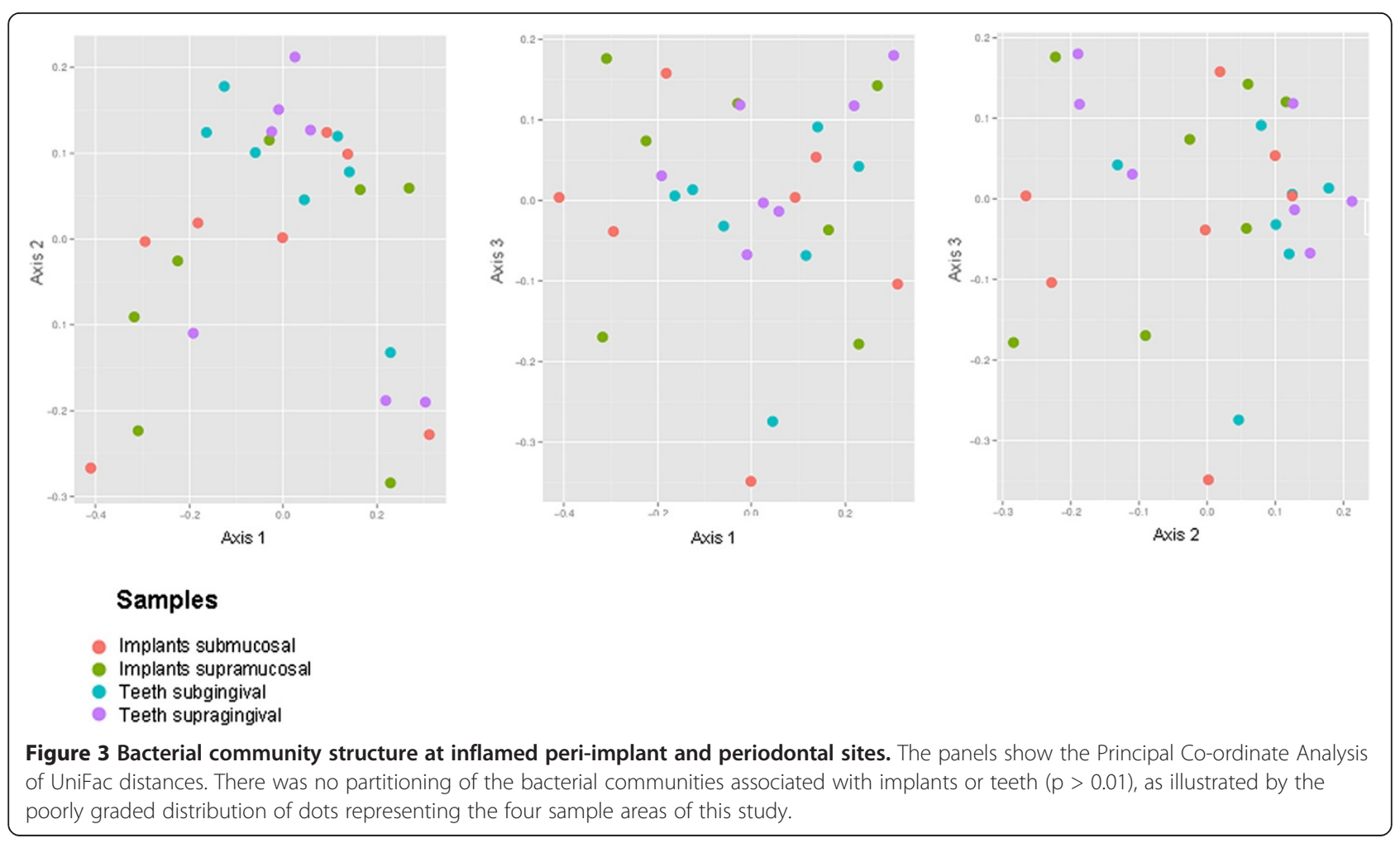




\section{Heat map}

Data visualization was performed using a heat map display, where the relative abundances of the 50 most frequent genera are represented by different brightnesses (Figure 4). Samples from different locations within individual patients shared only minimal similarities in bacterial community compositions, as shown with hierarchical clustering of bacterial taxa in the heat map display. Communities from supramucosal locations at implants closely clustered with communities from submucosal locations at implants. In contrast, samples taken from supragingival plaque were less similar to subgingival plaque samples at teeth.

\section{Shannon diversity index}

The Shannon Diversity index describes the biodiversity and considers the number of genera and their abundances [22]. Neither implants nor teeth demonstrated significant differences in the diversity index for supra- and submucosal locations at implants and supra- or subgingival locations at teeth (Figure 5).

\section{Discussion}

The present study describes in detail the supra- and submucosal, and supra- and subgingival microbiomes of inflamed peri-implant and periodontal sites in single subjects using $16 \mathrm{~S}$ rRNA gene-based pyrosequencing. The current study demonstrated (1) frequent occurrence of members of the genus Rothia and members of the family Streptococcaceae at implants and teeth, (2) no significant differences between the microbiomes of diseased implants and teeth affected by periodontitis, (3) no significant differences between supra- and submucosal, or supra- and subgingival microbiomes.

The current 16S rRNA approach was aimed to detect the comprehensive composition of bacteria located at two different sites at implants and teeth. In the present study, the sequencing lengths were limited to $550 \mathrm{bp}$ and

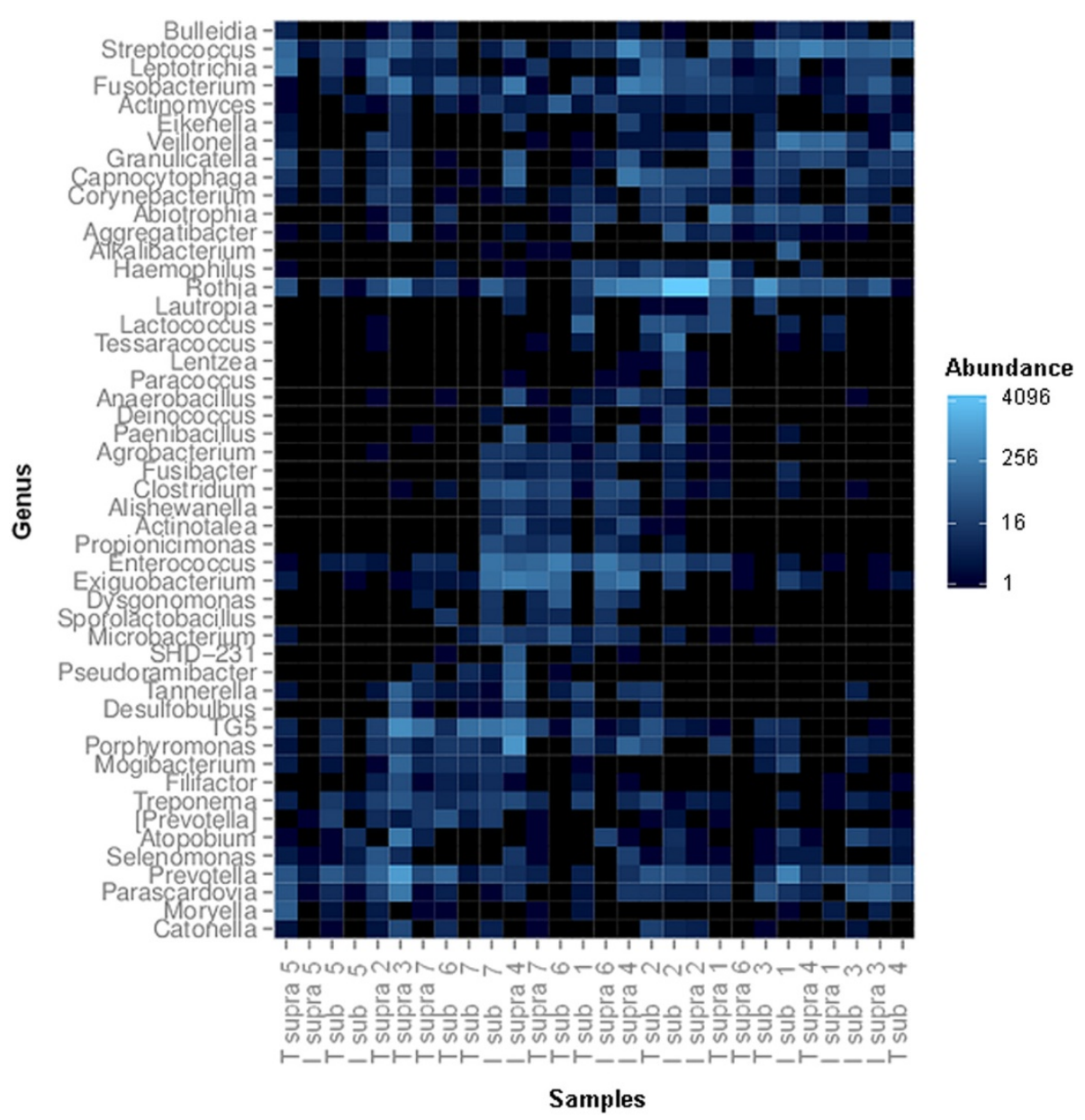

Figure 4 Heat map presentation showing the abundances of the $\mathbf{5 0}$ most frequent genera in all samples. Individual samples are depicted on the x-axis as tooth (T) or implant (I), the location supra (= supramucosal or supragingival) or sub (= submucosal or subgingival) and a number representing the patient. From this presentation, it is apparent that different locations within individual patients shared only minimal similarities in bacterial community compositions. 


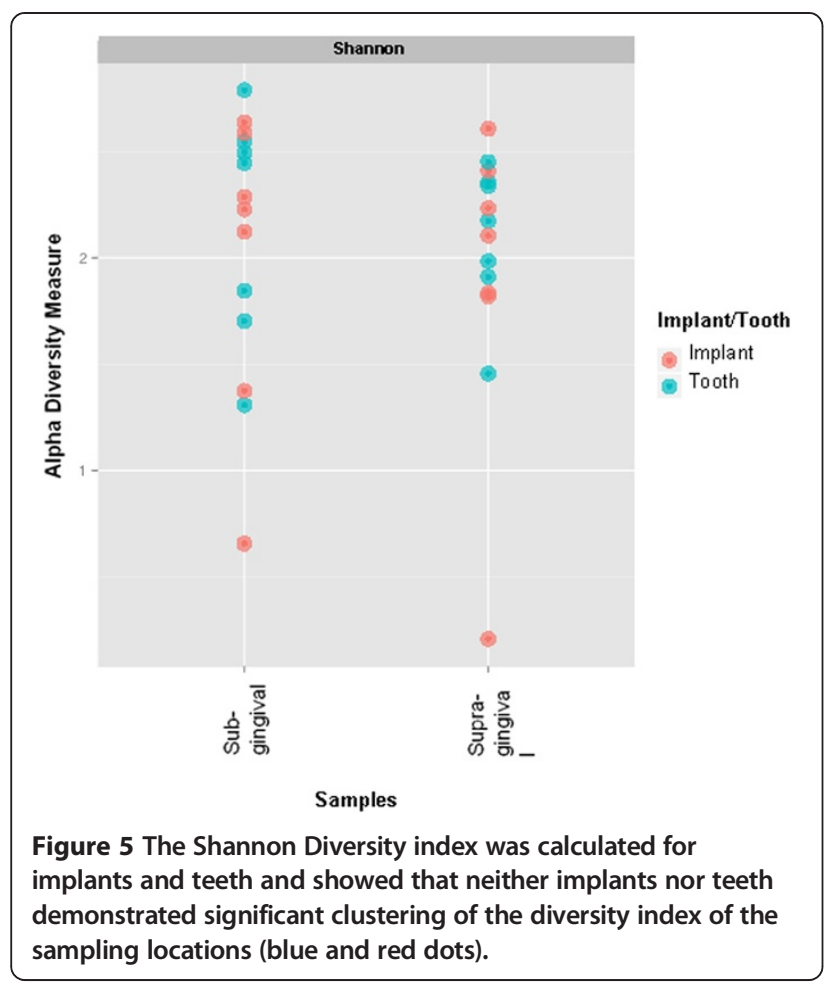

therefore annotations were restricted to the genus level, an established approach for the analysis of complex biofilms $[23,24]$. In agreement with other current publications, the composition of microbiomes showed high inter-individual differences [8]. Prominent phylotypes at supra- and submucosal regions were Rothia and Streptococcaceae. Species belonging to the genus Rothia have been repeatedly described as members of oral communities [25-27], and have been associated with periodontal health $[28,29]$. High levels of this genus have been reported at healthy implant sites as well [30]. Specific members of the genus Rothia have been recently shown to cause clinical infections such as septic arthritis, pneumonia, septicemia in renal transplant patients, arteriovenous infections, acute bronchitis and endocarditis [31] and - as a member of biofilms - has been associated with joint infections in orthopedics [32]. The virulence factors and the capacity of this genus to induce infections have been studied in vitro as well [33]. Our study also detected high frequencies of genera that have not been previously described as common oral inhabitants [34]. E.g. Exiguobacterium has been described as a bacterium colonizing marine habitats and sea food [35-37], ancient Siberian permafrost, Greenland glacial ice, and hot springs [38]. From the present study, it is unclear if this genus was accidentally incorporated by contamination [39] or if it was incorporated in oral plaques by food consumption., Food intake should therefore be accurately controlled or recorded in future studies.
All analyses in the present study indicated that the diversity of biofilms colonizing diseased implants was similar to biofilms colonizing teeth affected by periodontitis. In contrast, Kumar et al. [7] observed reduced diversity at implant sites than at diseased teeth and Koyanagi et al. [8] reported significantly higher diversity at implant sites than at diseased teeth. A partial explanation for these differences may be that the subjects were from different ethnic populations. It was hypothesized that diversity is an indicator of the complexity of a disease, whereas high diversity is associated with complex diseases.

In the present study, bacterial genera associated with diseased implants were not significantly different from communities associated with infected teeth in the same subject, which is in accordance with other publications [40-42] and demonstrated that the intraoral transmission of bacteria from one niche to the other is a feasible event. In contrast, with hybridization techniques the genus Actinomyces was the most dominant taxon found at teeth affected by periodontitis and diseased implants $[3,43]$, but was only found in low frequencies in the present study. Kumar et al. [7] used sequencing techniques and concluded that Actinomyces bacteria make up less than $5 \%$ of all sequences. The genera Treponema and Tannerella including species belonging to the red complex, as well as Aggregatibacter, were found in nearly similar frequencies at diseased implants and teeth affected by periodontitis; in contrast Porphyromonas was found more frequently at implants. The same observations were reported earlier by Cortelli et al. [44] but were not supported by other studies $[7,8]$. Again, differences in the experimental design may account for these observations, e.g. Kumar et al. [7] investigated implants and teeth from different subjects.

In our study, the compositions of supra- or submucosal biofilms at implants were more similar than the supra- or subgingival biofilms at teeth, as demonstrated by the heat map analysis, which is in accordance to Ximenez-Fyvie et al. [43] who found identical genera in supra- and subgingival plaques of teeth affected by periodontitis. Utilizing DNA hybridization, Shibli et al. [3] also confirmed the similarities between biofilms at supra- and submucosal locations at implants.

At implant sites, the microbial composition was mainly composed of Gram-positive taxa. At teeth, Gram-positive taxa were also more frequent than Gram-negative taxa, but at much lower ratios. These differences between supra- and submucosal locations were not obvious on discrimination of sequenced genera, but became obvious using Gram characteristics. These data are partially in contrast to data reported by Kumar et al. [7], who stated that peri-implantitis of failing implants is a predominantly Gram-negative disease. 


\section{Conclusions}

The present study using $16 \mathrm{~S}$ rRNA sequencing techniques complemented the knowledge of the composition of supraand submucosal, and supra- and subgingal biofilms. Based on the limitations of the study and the analysis on a genus level significant differences in the biofilm composition of diseased peri-implant and periodontal tissues were not observed.

\section{Additional file}

\section{Additional file 1: 16S rRNA gene sequences.}

\section{Abbreviations}

API: Approximal Plaque Index; BOP: Bleeding on probing; Bp: Base pair; DNA: Desoxyribonucleic acid; dNTP: Desoxynucleotide triphosphate; min.: Minute; ml: Milliliter; mm: Millimeter; no: Number; OUT: Operational taxonomic units; PCoA: Principal Coodinate Analyses; PCR: Polymerase Chain Reaction; PD: Pocket depth; rRNA: Ribosomal ribonucleic acid.

\section{Competing interests}

The authors declare that they have no competing interests.

\section{Authors' contribution}

SS contributed with concept and design, clinical investigation, analysis of data, and was responsible for drafting; IS clinical investigation; RS analysis of data; MaS sequencing; AW and SNS sample preparation; JE and MeS concept and design critically revised and approved the final version of the manuscript.

\section{Acknowledgements}

The authors are grateful to Rainer Schreeb for excellent laboratory work The study was funded in part by the Dr. Dorka-Stiftung.

\section{Author details}

${ }^{1}$ Department of Prosthetic Dentistry and Biomedical Materials Science, Hannover Medical School, Hannover, Germany. ${ }^{2}$ Department of Conservative Dentistry, Periodontology and Preventive Dentistry, Hannover Medical School, Hannover, Germany. ${ }^{3}$ Institute for Biometry, Hannover Medical School, Hannover, Germany. ${ }^{4}$ Institute of Clinical Molecular Biology, Christian-Albrechts-University Kiel, Kiel, Germany. ${ }^{5}$ Peri-implant and Oral Infections, Department of Prosthetic Dentistry and Biomedical Materials Science, Hannover Medical School, Carl-Neuberg-Strasse 1, 30625 Hannover, Germany.

Received: 28 August 2014 Accepted: 15 December 2014 Published: 17 December 2014

\section{References}

1. Mombelli A, Muller N, Cionca N: The epidemiology of peri-implantitis. Clin Oral Implants Res 2012, 23(Suppl 6):67-76.

2. Heitz-Mayfield LJ: Peri-implant diseases: diagnosis and risk indicators. J Clin Periodontol 2008, 35(8 Suppl):292-304.

3. Shibli JA, Melo L, Ferrari DS, Figueiredo LC, Faveri M, Feres M: Composition of supra- and subgingival biofilm of subjects with healthy and diseased implants. Clin Oral Implants Res 2008, 19(10):975-982.

4. Persson GR, Samuelsson E, Lindahl C, Renvert S: Mechanical non-surgical treatment of peri-implantitis: a single-blinded randomized longitudinal clinical study. II. Microbiological results. J Clin Periodontol 2010, 37(6):563-573.

5. Maximo MB, de Mendonca AC, Renata Santos V, Figueiredo LC, Feres M, Duarte PM: Short-term clinical and microbiological evaluations of peri-implant diseases before and after mechanical anti-infective therapies. Clin Oral Implants Res 2009, 20(1):99-108.

6. Salcetti JM, Moriarty JD, Cooper LF, Smith FW, Collins JG, Socransky SS, Offenbacher S: The clinical, microbial, and host response characteristics of the failing implant. Int J Oral Maxillofac Implants 1997, 12(1):32-42.
7. Kumar PS, Mason MR, Brooker MR, O'Brien K: Pyrosequencing reveals unique microbial signatures associated with healthy and failing dental implants. J Clin Periodontol 2012, 39(5):425-433.

8. Koyanagi T, Sakamoto M, Takeuchi Y, Maruyama N, Ohkuma M, Izumi Y: Comprehensive microbiological findings in peri-implantitis and periodontitis. J Clin Periodontol 2013, 40(3):218-226.

9. Dabdoub SM, Tsigarida AA, Kumar PS: Patient-specific analysis of periodontal and peri-implant microbiomes. J Dent Res 2013, 92(12 Suppl):168S-175S.

10. Hellstrom MK, Ramberg P, Krok L, Lindhe J: The effect of supragingival plaque control on the subgingival microflora in human periodontitis. J Clin Periodontol 1996, 23(10):934-940.

11. Socransky SS, Haffajee AD: Dental biofilms: difficult therapeutic targets. Periodontol 2000 2002, 28:12-55.

12. Tezal M, Scannapieco FA, Wactawski-Wende J, Grossi SG, Genco RJ: Supragingival plaque may modify the effects of subgingival bacteria on attachment loss. J Periodontol 2006, 77(5):808-813.

13. Teughels W, Van Assche N, Sliepen I, Quirynen M: Effect of material characteristics and/or surface topography on biofilm development. Clin Oral Implants Res 2006, 17(Suppl 2):68-81.

14. Heitz-Mayfield LJ, Lang NP: Comparative biology of chronic and aggressive periodontitis vs. peri-implantitis. Periodontol 2000 2010, 53:167-181.

15. Lange DE, Plagmann H-C, Eenboom A, Promesberger A: Klinische Bewertungsverfahren zur Objektivierung der Mundhygiene. Dtsch zahnärztl Z 1977, 32:44-47.

16. Caporaso JG, Kuczynski J, Stombaugh J, Bittinger K, Bushman FD, Costello EK, Fierer N, Pena AG, Goodrich JK, Gordon JI, Huttley GA, Kelley ST, Knights D, Koenig JE, Ley RE, Lozupone CA, McDonald D, Muegge BD, Pirrung M, Reeder J, Sevinsky JR, Turnbaugh PJ, Walters WA, Widmann J, Yatsunenko T, Zaneveld J, Knight R: QIIME allows analysis of high-throughput community sequencing data. Nat Methods 2010, 7(5):335-336.

17. Reeder J, Knight R: Rapidly denoising pyrosequencing amplicon reads by exploiting rank-abundance distributions. Nat Methods 2010, 7(9):668-669.

18. R Core Team: $R$ : A language and environment for statistical computing. Vienna, Austria: R Foundation for Statistical Computing; 2013. http://www.R-project.org

19. Gentleman RC, Carey VJ, Bates DM, Bolstad B, Dettling M, Dudoit S, Ellis B, Gautier L, Ge Y, Gentry J, Hornik K, Hothorn T, Huber W, lacus S, Irizarry R, Leisch F, Li C, Maechler M, Rossini AJ, Sawitzki G, Smith C, Smyth G, Tierney L, Yang JY, Zhang J: Bioconductor: open software development for computational biology and bioinformatics. Genome Biol 2004, 5(10):R80

20. McMurdie PJ, Holmes S: phyloseq: an R package for reproducible interactive analysis and graphics of microbiome census data. PLoS One 2013, 8(4):e61217.

21. Robinson MD, McCarthy DJ, Smyth GK: edgeR: a Bioconductor package for differential expression analysis of digital gene expression data. Bioinformatics 2010, 26(1):139-140.

22. Shannon CE: The mathematical theory of communication. MD Comput 1997 1963, 14(4):306-317.

23. Bizzarro S, Loos BG, Laine ML, Crielaard W, Zaura E: Subgingival microbiome in smokers and non-smokers in periodontitis: an exploratory study using traditional targeted techniques and a next-generation sequencing. J Clin Periodontol 2013, 40(5):483-492.

24. Hu YJ, Wang Q, Jiang YT, Ma R, Xia WW, Tang ZS, Liu Z, Liang JP, Huang ZW: Characterization of oral bacterial diversity of irradiated patients by high-throughput sequencing. Int J Oral Sci 2013, 5(1):21-25.

25. Tanner AC, Haffer C, Bratthall GT, Visconti RA, Socransky SS: A study of the bacteria associated with advancing periodontitis in man. $J$ Clin Periodontol 1979, 6(5):278-307.

26. Lesher RJ, Gerencser VF, Morrison DJ: Presence of Rothia dentocariosa strain 477 serotype 2 in gingiva of patients with inflammatory periodontal disease. J Dent Res 1977, 56(2):189.

27. Segata N, Haake SK, Mannon P, Lemon KP, Waldron L, Gevers D, Huttenhower C, Izard J: Composition of the adult digestive tract bacterial microbiome based on seven mouth surfaces, tonsils, throat and stool samples. Genome Biol 2012, 13(6):R42.

28. Kistler JO, Booth V, Bradshaw DJ, Wade WG: Bacterial community development in experimental gingivitis. PLoS One 2013, 8(8):e71227.

29. Abusleme L, Dupuy AK, Dutzan N, Silva N, Burleson JA, Strausbaugh LD, Gamonal J, Diaz PI: The subgingival microbiome in health and periodontitis and its relationship with community biomass and inflammation. ISME J 2013, 7(5):1016-1025. 
30. da Silva ES, Feres M, Figueiredo LC, Shibli JA, Ramiro FS, Faveri M: Microbiological diversity of peri-implantitis biofilm by Sanger sequencing. Clin Oral Implants Res 2014, 25(10):1192-1199.

31. Shakoor S, Fasih N, Jabeen K, Jamil B: Rothia dentocariosa endocarditis with mitral valve prolapse: case report and brief review. Infection 2011, 39(2):177-179.

32. Trivedi MN, Malhotra P: Rothia prosthetic knee joint infection. J Microbiol Immunol Infect 2013, 25:243-245.

33. Kataoka $\mathrm{H}$, Taniguchi $\mathrm{M}$, Fukamachi $\mathrm{H}$, Arimoto $T$, Morisaki $\mathrm{H}$, Kuwata $\mathrm{H}$ : Rothia dentocariosa induces TNF-alpha production in a TLR2-dependent manner. Pathog Dis 2014, 71(1):65-68.

34. Chen T, Yu WH, Izard J, Baranova OV, Lakshmanan A, Dewhirst FE: The Human Oral Microbiome Database: a web accessible resource for investigating oral microbe taxonomic and genomic information. Database (Oxford) 2010, 2010:baq013.

35. Inbakandan D, Murthy PS, Venkatesan R, Khan SA: $16 S$ rDNA sequence analysis of culturable marine biofilm forming bacteria from a ship's hull. Biofouling 2010, 26(8):893-899.

36. Wen W, Wang S, Zhou X, Fang B: Central carbon metabolism in marine bacteria examined with a simplified assay for dehydrogenases. Appl Biochem Biotechnol 2013, 170(3):473-482.

37. Yang J, Wang C, Wu J, Liu L, Zhang G, Feng J: Characterization of a multi-resistant mosaic plasmid from a fish farm sediment Exiguobacterium sp. isolate reveals aggregation of functional clinically-associated antibiotic resistance genes. Appl Environ Microbiol 2014, 80(4):1482-1488.

38. Vishnivetskaya TA, Kathariou S, Tiedje JM: The Exiguobacterium genus: biodiversity and biogeography. Extremophiles 2009, 13(3):541-555.

39. van der Horst J, Buijs MJ, Laine ML, Wismeijer D, Loos BG, Crielaard W, Zaura E: Sterile paper points as a bacterial DNA-contamination source in microbiome profiles of clinical samples. J Dent 2013, 41(12):1297-1301.

40. Sumida S, Ishihara K, Kishi M, Okuda K: Transmission of periodontal disease-associated bacteria from teeth to osseointegrated implant regions. Int J Oral Maxillofac Implants 2002, 17(5):696-702.

41. Quirynen M, Papaioannou W, van Steenberghe D: Intraoral transmission and the colonization of oral hard surfaces. J Periodontol 1996, 67(10):986-993.

42. Quirynen M, Vogels R, Peeters W, van Steenberghe D, Naert I, Haffajee A: Dynamics of initial subgingival colonization of 'pristine' peri-implant pockets. Clin Oral Implants Res 2006, 17(1):25-37.

43. Ximenez-Fyvie LA, Haffajee AD, Socransky SS: Microbial composition of supra- and subgingival plaque in subjects with adult periodontitis. J Clin Periodontol 2000, 27(10):722-732.

44. Cortelli SC, Cortelli JR, Romeiro RL, Costa FO, Aquino DR, Orzechowski PR, Araujo VC, Duarte PM: Frequency of periodontal pathogens in equivalent peri-implant and periodontal clinical statuses. Arch Oral Biol 2013, 58(1):67-74

doi:10.1186/1472-6831-14-157

Cite this article as: Schaumann et al:: Pyrosequencing of supra- and subgingival biofilms from inflamed peri-implant and periodontal sites. BMC Oral Health 2014 14:157.

\section{Submit your next manuscript to BioMed Central and take full advantage of:}

- Convenient online submission

- Thorough peer review

- No space constraints or color figure charges

- Immediate publication on acceptance

- Inclusion in PubMed, CAS, Scopus and Google Scholar

- Research which is freely available for redistribution 